\title{
Comparative Morphometric Analysis for Differentiation of Three Demodex Mite Species causing Canine Demodicosis
}

\author{
Alok Kumar Chaudhary*, Umesh Dimri, Y. Ajith, E. Madesh, \\ Supriya Yadav, K. Kavitha and Stanzin Angmo
}

\author{
Division of Veterinary Medicine Indian Veterinary Research Institute, \\ Izatnagar, Bareilly, India (UP) \\ *Corresponding author
}

\section{A B S T R A C T}

\section{Keywords}

Demodex canis, $D$. cornei and D. injai, micrometry, Canine demodicosis

Article Info

Accepted:

16 November 2020

Available Online:

10 December 2020
In the present study, Demodex mite species of dog were differentiated based on their morphometric analysis. For mite's micrometry, skin scarping materials were collected from 40 demodicosis clinically infected dogs, presented with different clinical manifestations. Out of 150 dog mite's species $D$. canis $(\mathrm{n}=50), D$. cornei $(\mathrm{n}=50)$ and D. injai $(\mathrm{n}=50)$ were randomly selected for ocular micrometry of mites, body size and its segments. Correlation between body size and its body segments of all three Demodex species were estimated by descriptive statistical data analysis. There was a significant correlation between total body length and length of the podosoma and opisthosoma $(\mathrm{p}<0.05)$ of $D$. injai as compared to D. canis and D. cornei species of dog mites.

\section{Introduction}

Demodex canis specie was first discovered demodex mite specie of dog by Simon in 1844. Morphological description and name was given by Leydig in 1859. Demodex injai was first reported by Desch and Hillier in 2003 and Demodex cornei, was firstly discovered by Mason in the 20th century. Based on mitochondrial marker $16 \mathrm{~S}$ rDNA and Cytochrome Oxidase- I, study of Rojas et al., (2012) concluded that, remaining two forms of reported dog mite Demodex injai and Demodex cornei are polymorphs of the
Demodex canis species. Remarkable study of Sastre et al., (2012) who analysed phylogenetic trait of these three species based on partial sequence of mitochondrial 16rDNA and proposed that, Demodex injai is the separate species of dog mite and latter on Milosevic et al., (2013) confirmed that, Demodex injai are the valid separate species of dogs mites. Family Demodicidae, have a small, thin, usually elongated body, with four pairs of legs. Their bodies are divided into three distinct part called as gnathosoma (mouth and head part), podosoma consists of four pairs of legs and opisthosoma part of 
elongated tail. All the three demodex mites species have been reported in India as well as abroad Veena et al., 2017 and Fathima et al., 2017. The present investigation was aimed to study the morphometry of three Demodex mites, i.e. D. canis, D. injai and D. cornei in dogs with demodicosis. It might be helpful in the identification of mite species based on their morphology and its measurements.

\section{Materials and Methods}

Identification and morphometric measurements were performed in clinical laboratory of Division of Medicine, ICARIndian Veterinary Research Institute, Izatnagar, UP, India. The exact size of a microorganism was determined by using calibrated ocular lens and stage micrometer (Erma objective micrometer, Japan) under compound light microscope (Olympus microscope Model CH-20i.) as per method described by Gonzale and Bendall, (1995) and mites were identified on the basis of morphological characteristics as described by Soulsby, (1996). All the ratios were calculated by using simple arithmetic formulas.

\section{Results and Discussion}

Micrometry of 150 mites revealed that, mean length of total body of Demodex injai species was $263.610 \pm 2.83^{\text {c. }}$ It was significantly larger as compared to $D$. canis and D. cornei species of mites $(\mathrm{p}<0.05)$. It was more or less similar to study of Swathi et al., 2016 who reported the mean body length for Demodex injai was $264 \pm 6.89 \mu$. Maximum mean length for Demodex injai has been measured by Desch et al., 2003 it was found that, $361.3 \pm$ $43.9 \mu \mathrm{m}$ of mean length of total body. Among three demodex species, Demodex cornei was shorter in mean length of total body (156.887 $\left.\pm 1.35^{\mathrm{b}} \mu \mathrm{m}\right)$. Recently, some researchers was also reported shorter mean length of same mites species, Sakulpoy and Sangvaranond 2010 found to be very closely with finding of the present study $(156.92 \mu \mathrm{m})$ and other were also agreed with similar findings Fathima et al.,2017 and Swathi, et al., 2016. The mean length of total body of Demodex canis was $223.822 \pm 1.69^{\mathrm{a}} \mu \mathrm{m}$. It was larger as compared to Demodex cornei body length but shorter to Demodex injai. It was matched with the findings of ${ }^{[11]}$, who had reported mean body lengths of Demodex canis as, $211.81 \pm$ 14.86 and $214.32 \pm 13.81 \mu \mathrm{m}$, respectively.

Body segments of all three mite species were also showed significant difference. The mean length/width of first segments of body (gnathosoma) of $D$. canis, D. injai and $D$. cornei were $21.76 \pm 0.29^{\mathrm{a}} / 20.49 \pm 0.32^{\mathrm{c}}$, $18.85 \pm 0.64^{\mathrm{b}} \mu \mathrm{m} / 16.83 \pm 0.88^{\mathrm{b}} \mu \mathrm{m}$ and 22.42 $\pm 0.60^{\mathrm{a}} \mu \mathrm{m} / 24.04 \pm 0.70^{\mathrm{c}} \mu \mathrm{m}$ respectively. Mean length and width of gnathosoma in Demodex cornei was significantly shorter and wider as compared to $D$. canis and of Demodex injai gnathosoma. It agrees with findings of Fathima et al., 2017 and Swathi, et al., 2016.

Mean length/width of second segments of body (podosoma) of $D$. canis, $D$. injai and $D$. cornei were $70.38 \pm 1.05 \mathrm{a} / 39.02 \pm 0.42^{\mathrm{a}}$, $74.94 \pm 0.77^{\mathrm{c}} / 43.03 \pm 0.63^{\mathrm{c}}$ and $56.89 \pm 0.90$ ${ }^{\mathrm{b}} / 23.91 \pm 0.90^{\mathrm{b}}$ respectively. Among these larger length of podosoma was measured in Demodex injai. In contrast to, shorter length and comparatively broader width of podosoma were measured in D. cornei. It was agreed with Desch and Nutting 1998.

Significant variation was noticed in length and width of opisthosoma (third body segment) in all three reported species of demodex. Larger opisthosoma was observed in $D$. injai with mean length $166.246 \pm 2.55^{\mathrm{c}}$ $\mu \mathrm{m}$ and width $37.552 \pm 4.39^{\mathrm{a}} \mu \mathrm{m}$. Blunt and shorter opisthosoma was noticed in $D$. cornei species as $81.137 \pm 1.03^{\mathrm{b}} \mu \mathrm{m}$ and width was 
$24.24 \pm 0.9^{\mathrm{a}} \mu \mathrm{m}$ and in D. canis species, it was medium in length $131.674 \pm 1.20{ }^{\mathrm{a}} \mu \mathrm{m}$ and width $34.8 \pm 0.52 \mathrm{a} \mu \mathrm{m}$. It was similar to those findings of Desch and Nutting 1998 (Fig. 1-4).

Table.1 Micrometric analysis of mite's species of dog causing demodicosis

\begin{tabular}{|c|c|c|c|c|c|c|}
\hline \multirow[t]{2}{*}{ Part of body } & \multirow{2}{*}{\multicolumn{2}{|c|}{ Statistics }} & D. canis & D.cornai & D. injai & $P$ value \\
\hline & & & $\mathrm{N}=110$ & $\mathrm{~N}=30$ & \multirow{2}{*}{$\begin{array}{c}N=50 \\
22.42 \pm 0.60^{a}\end{array}$} & \multirow[t]{4}{*}{$<0.05$} \\
\hline \multirow[t]{6}{*}{ Gnathosoma $(\mu \mathrm{m})$} & \multirow[t]{3}{*}{$\mathrm{L}$} & Mean & $21.76 \pm 0.29^{\mathrm{a}}$ & $18.85 \pm 0.64^{\mathrm{b}}$ & & \\
\hline & & Range & $27.27-10.10$ & $20.20-10.10$ & $30.30-20.20$ & \\
\hline & & G.mean & 21.51 & 18.42 & 22.08 & \\
\hline & W & Mean & $20.49 \pm 0.32^{\mathrm{a}}$ & $16.83 \pm 0.88^{b}$ & $24.04 \pm 0.70^{c}$ & $<0.05$ \\
\hline & & Range & $28.00-12.00$ & $20.20-10.10$ & $30.30-20.20$ & \\
\hline & & G.mean & 20.19 & 16.03 & 23.56 & \\
\hline \multirow[t]{6}{*}{ Podosoma $(\mu \mathrm{m})$} & \multirow[t]{3}{*}{$\mathrm{L}$} & Mean & $70.38 \pm 1.05^{\mathrm{a}}$ & $56.89 \pm 0.90^{b}$ & $74.94 \pm 0.77^{\mathrm{c}}$ & \multirow{3}{*}{$<0.05$} \\
\hline & & Range & $90.90-50.50$ & $60.60-50.50$ & $90.90-70.70$ & \\
\hline & & G.mean & 69.51 & 56.68 & 74.75 & \\
\hline & \multirow[t]{3}{*}{$\mathrm{W}$} & Mean & $39.02 \pm 0.42^{\mathrm{a}}$ & $23.91 \pm 0.90^{b}$ & $43.03 \pm 0.63^{c}$ & \multirow[t]{3}{*}{$<0.05$} \\
\hline & & Range & $40.40-20.20$ & $30.3-020.20$ & $50.50-40.40$ & \\
\hline & & G.mean & 38.68 & 23.44 & 42.81 & \\
\hline \multirow{6}{*}{$\begin{array}{l}\text { Opisthosoma } \\
(\mu \mathrm{m})\end{array}$} & \multirow[t]{3}{*}{$\mathrm{L}$} & Mean & $131.674 \pm 1.20^{\mathrm{a}}$ & $81.137 \pm 1.03^{b}$ & $166.246 \pm 2.55^{c}$ & \multirow[t]{3}{*}{$<0.05$} \\
\hline & & Range & $161.60-101.00$ & $90.90-70.70$ & $212.10-141.40$ & \\
\hline & & G.mean & 131.08 & 80.95 & 165.32 & \\
\hline & \multirow[t]{3}{*}{ W } & Mean & $34.8 \pm 0.52^{\mathrm{a}}$ & $24.24 \pm 0.92^{\mathrm{a}}$ & $37.55 \pm 4.39^{a}$ & \multirow[t]{3}{*}{$>0.05$} \\
\hline & & Range & $50.50-30.30$ & $30.30-20.20$ & $43.43-19.19$ & \\
\hline & & G.mean & 34.49 & 23.76 & 26.61 & \\
\hline \multirow{3}{*}{$\begin{array}{l}\text { Total body length } \\
(\mu \mathrm{m})\end{array}$} & $\mathrm{L}$ & Mean & $223.822 \pm 1.69^{\mathrm{a}}$ & $156.887 \pm 1.35^{b}$ & $263.610 \pm 2.83^{c}$ & \multirow[t]{3}{*}{$<0.05$} \\
\hline & & Range & 266.64-175.74 & $171.70-141.40$ & $313.10-232.30$ & \\
\hline & & G.mean & 223.12 & 156.72 & 262.88 & \\
\hline \multicolumn{7}{|c|}{ Differences in divisions, fraction and ratios of mites species body segments } \\
\hline \multirow[t]{3}{*}{ Ratio of G: TB } & & Division & 0.097 & 0.120 & \multicolumn{2}{|c|}{0.085} \\
\hline & & Fraction & $7 / 72$ & $3 / 25$ & \multicolumn{2}{|c|}{$4 / 47$} \\
\hline & & Ratio & 07: 72 & 03:25 & \multicolumn{2}{|c|}{$04: 47$} \\
\hline \multirow[t]{3}{*}{ Ratio of P:TB } & & Division & 0.314 & 0.363 & \multicolumn{2}{|c|}{0.284} \\
\hline & & Fraction & $11 / 35$ & $33 / 91$ & $27 / 9$ & \\
\hline & & Ratio & $11: 35$ & $33: 91$ & $27: 9$ & \\
\hline Ratio of O: TB & & Division & 0.588 & 0.517 & 0.63 & \\
\hline & & Fraction & $10 / 17$ & $15 / 29$ & $41 / 6$ & \\
\hline & & Ratio & $10: 17$ & $15: 29$ & $41: 6$ & \\
\hline Ratio of G:P & & Division & 0.309 & 0.331 & 0.29 & \\
\hline & & Fraction & $17 / 55$ & $1 / 3$ & $3 / 10$ & \\
\hline & & Ratio & $17: 55$ & 01:03 & 03:1 & \\
\hline Ratio of P: O & & Division & 0.535 & 0.701 & 0.45 & \\
\hline & & Fraction & $31 / 58$ & $61 / 87$ & $32 / 7$ & \\
\hline & & Ratio & $31: 58$ & $61: 87$ & $32: 7$ & \\
\hline
\end{tabular}


Fig.1 Adult Demodex canis, Fig.2 Adult Demodex injai, Fig.3 Adult Demodex cornei and Fig.4 Gnathosoma (first body segment) and podosoma of adult Demodex canis

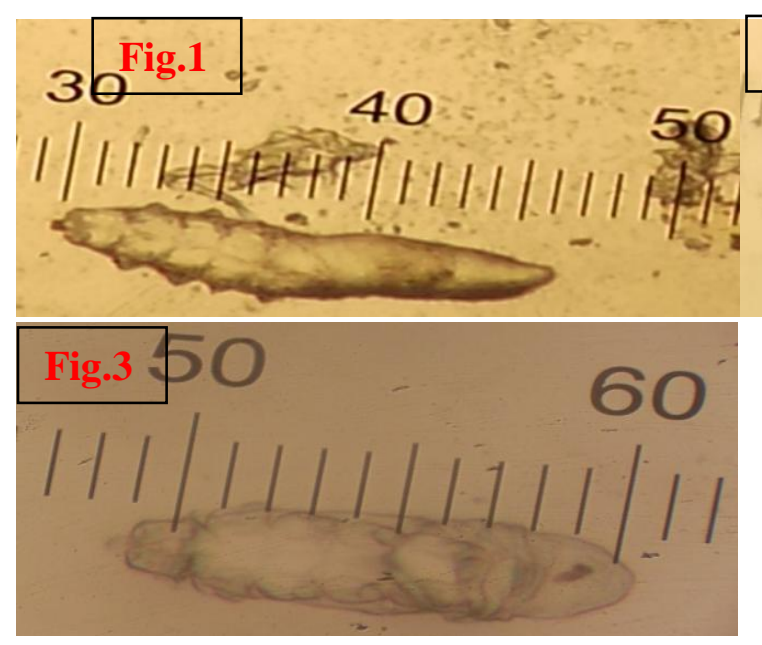

Calculated ratios of Gnathosoma length mean and total body length mean of Demodex canis it was 07: 72, in Demodex cornei was 03:25 and in Demodex injai was 04:47. Ratios of Podosoma length mean and total body length mean (P: TB) of D. canis was 11:35 in $D$. cornei was 33:91 and in Demodex injai was 27:95.

Ratios of opisthosoma length mean and total body length mean (O: TB) of $D$. canis was 10:17 in D. cornei was 15:29and in Demodex injai was 41:65.The ratio between gnathosoma and opisthosoma (G: O) length in of $D$. canis was $17: 55$ in $D$. corne $i$ was $1: 3$ and in Demodex injai was 3:10. The ratio between podosoma and opisthosoma (P: O) length in D. canis was $31: 58$, in D. cornei was $61: 87$ and in Demodex injai was 32:7. Calculated ratios were similar to findings of Swathi, et al., 2016.

In conclusion three species of demodex mite were observed in the study viz., Demodex injai, D. canis and D. cornei. The morphometry of mites revealed that mean total body length of Demodex cornei was much less than that of Demodex canis and Demodex injai. D. cornei had short opisthosoma and blunted posterior end as

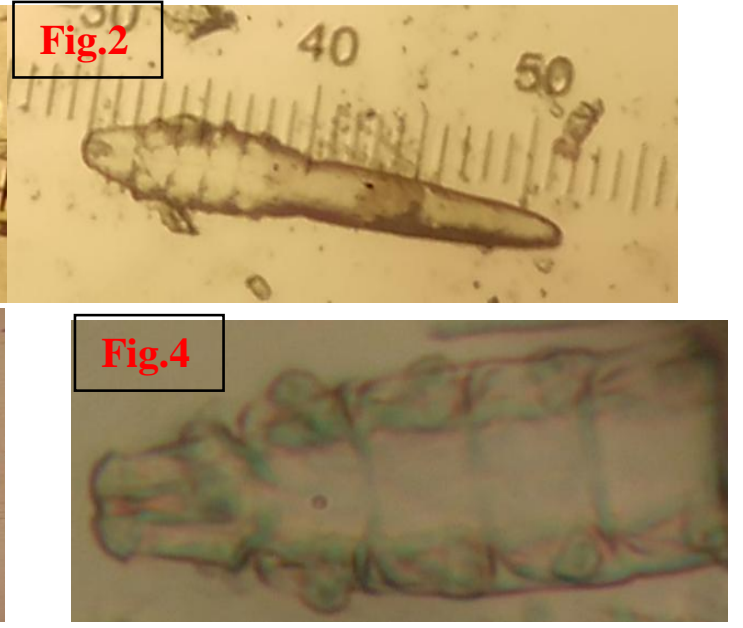

compared to other remaining species of dog's demodex mite. Demodex injai had tall and thinner opisthosoma with pointed end. Calculated ratios indicate that approximated relationship within or between species of body segments of Demodex mites of dog.

\section{Acknowledgements}

Authors are thankful to Clinical lab of Medicine Division, IVRI, and India for providing facilities.

\section{References}

Chen C: A short-tailed demodectic mite and demodex infestation in a Chihuahua dog. Vet Dermatol 1995, (6): 227-229.

Desch, C., and Nutting W: Demodex canis redescription and reevaluation. Cornell Vet: 1978; (68): 139 Đ149.

Fathima L, Sreenivasamurthy GS and Udayakumar M: Morphometry of demodex species of canines in Hyderabad region of Telangana state: Journal of Entomology and Zoology Studies 2017; 5(6): 1776-1779.

González- A., and Bendall, R. P. Size matters: the use of the ocular micrometer in diagnostic parasitology. Parasitology 
Today: $1995 ; 11(2), 83-85$.

Hillier A, Desch CE: Large-bodied Demodex mite infestation in 4 dogs. J Am Vet Med Assoc: 2002; (220): 623-627

Leydig F: About hair follicle mites and itch mite. Arch Natur Berli: 1859; (1): 338. 8.

Mason KV: A new species of Demodex mite with D. canis causing canine demodicosis: a case report. Vet Dermatol: 1993; (4): 37. 12.

Milosevic MA, Frank LA, Brahmbhatt RA, Kania SA. PCR amplification and DNA sequencing of Demodex injai from otic secretions of a dog. Vet Dermatol. 2013; (24):286-e66.

Rojas D, Riazzo M, Callejón C, Guevara. and Cutillas, C. :Molecular study on three morphotypes of Demodex mites (Acarina: Demodicidae) from dogs. Parasitology research: 2012; 111(5), 2165-2172.

Sastre N, Ravera I, Villanueva S, Altet L, Bardagit M, Sánchez A, et al. Phylogenetic relationships in three species of canine Demodex mite based on partial sequences of mitochondrial
16 s rDNA. Vet Dermatol: 2012; (23): 509-e101.

Sivajothi S, Reddy BS, Kumari KN, Rayulu VC. Morphometry of Demodex canis and Demodex cornei in dogs with demodicosis in India. International Journal of Veterinary Health Science and Research. 2013; (1):301.

Soulsby, A., and Clark, E.: The emergence of post-communist management in the Czech Republic. Organization studies,:1996; 17(2), 227-247

Swathi, S. Ayodhya and K. Satishkumar: Micrometry for differentiation of demodex mite species causing canine demodicosis in India. 2016: Int. J. Adv. Res. 4(11), 726-731

Veena M, Dhanalakshmi H, Kavitha K, Placid ED' Souza and GC Puttalaksmamma: Morphological characterization of demodex mites and its therapeutic management with neem leaves in canine demodicosis: Journal of Entomology and Zoology Studies 2017; 5(5): 661-664.

\section{How to cite this article:}

Alok Kumar Chaudhary, Umesh Dimri, Y. Ajith, E. Madesh, Supriya Yadav, K. Kavitha and Stanzin Angmo. 2020. Comparative Morphometric Analysis for Differentiation of Three Demodex Mite Species causing Canine Demodicosis. Int.J.Curr.Microbiol.App.Sci. 9(12): 2151-2155. doi: https://doi.org/10.20546/ijcmas.2020.912.253 